\title{
Numerical Visualization of the Unsteady Shock Wave Flow Field in Micro Shock Tube
}

\author{
Arun Kumar R* and Heuy Dong $\mathrm{Kim}^{\dagger}$
}

\begin{abstract}
Recently micro shock tube is extensively being used in many diverse fields of engineering applications but the detailed flow physics involved in it is hardly known due to high Knudsen number and strong compressibility effects. Unlike the macro shock tube, the surface area to volume ratio for a micro shock tube is very large. This unique effect brings many complexities into the flow physics that makes the micro shock tube different compared with the macro shock tube. In micro shock tube, the inter- molecular forces of working gas can play an important role in specifying the flow characteristics of the unsteady shock wave flow which is essentially generated in all kinds of shock tubes. In the present study, a CFD method was used to predict and visualize the unsteady shock wave flows using the unsteady compressible Navier-Stokes equations, furnished with the no-slip and slip wall boundary conditions. Maxwell's slip equations were used to mathematically model the shock movement at high Knudsen number. The present CFD results show that the propagation speed of the shock wave is directly proportional to the initial pressure and diameter of micro shock tube.
\end{abstract}

Key Words: Shock Wave, Micro Shock Tube, Unsteady Flow, Slip Wall, Shock Wave Reflection, Shock Wave Propagation

\section{Nomenclature}

$\mathrm{k}$ : Turbulent kinetic energy $\left(\mathrm{m}^{2} / \mathrm{s}^{2}\right)$

kn: Knudsen number

M: Mach number

P: Static pressure $(\mathrm{Pa})$

$\mathrm{P}_{\mathrm{r}}$ : Prandtl number

T: Temperature (k)

t: Time (s)

$\mathrm{u}_{\mathrm{i}}$ : Velocity components $(\mathrm{m} / \mathrm{s})$

$\mathrm{U}$ : Average velocities $(\mathrm{m} / \mathrm{s})$

$\rho$ : Density $\left(\mathrm{Kg} / \mathrm{m}^{3}\right)$

$\alpha$ : Thermal conductivity (W/mk)

$\lambda$ : Molecular mean free path $(\mathrm{m})$

$\dagger$ Department of Mechanical Engineering, Andong National University, Korea

E-mail : kimhd@andong.ac.kr

*Department of Mechanical Engineering, Andong National University, Korea $\delta$ : First layer height $(\mathrm{m})$

$\tau_{\mathrm{ijj}}$ : Stress tensor

$\underline{\mathrm{ui}, \mathrm{u}_{\mathrm{i}}^{1}}$ : Mean and fluctuating components $(\mathrm{m} / \mathrm{s})$

$\overline{\mathrm{u}_{1}^{\mathrm{J}}, \mathrm{u}_{\mathrm{j}}}:$ Reynolds-stress tensor

$\mu_{1}$ : Turbulent viscosity $(\mathrm{kg} / \mathrm{ms})$

$\mu$ : Dynamic viscosity (Pa s)

$\mu_{\text {eff: }}$ Effective viscosity $(\mathrm{kg} / \mathrm{ms})$

$\gamma$ : Ratio of specific heat

$\omega$ : Specific dissipation rate $\left(\mathrm{m}^{2} / \mathrm{s}^{2}\right)$

$\mathrm{D}_{\omega}$ : Cross diffusion Terms

$\mathrm{G}_{\mathrm{k}}, \mathrm{G}_{\omega}$ : Generation of $\mathrm{k}$ and $\omega$

$\Gamma_{k}, \Gamma_{\omega}$ : Effective diffusivity of $\mathrm{k}$ and $\omega$

\section{Subscripts}
1: Driven
4: Driver section
$c$ : Cell center quantities
$g$ : Gas quantities
$w$ : Wall quantities 


\section{Introduction}

Past few years have witnessed the growing importance of micro scale devices in various engineering applications like micro-electro mechanical systems, micro heat engines, micro propulsion devices, particle delivery devices etc. Most of these equipments require high speed or high temperature flow field to meet its operational requirements. For example, combustion within a micro heat engine ${ }^{(1)}$ requires the generation of high temperature flow field within a short period of time and within a constricted space. Another application, which is a hand held particle delivery system ${ }^{(2)}$ used to inject drug particles, requires a high flow speed to impart sufficient momentum to the particles. One of the preeminent equipment which can provide these extreme flow conditions required for the operation of such small scale devices is a micro shock tube. In general, a shock tube generates moving shock waves which propagates at very high speed and produce sudden increase in temperature.

In the past, the shock characteristics inside a macro shock tube have been well established through various experimental and theoretical works. The working principle and physical features of micro shock tube are similar as that of a conventional macro shock tube except its low flow dimension. Such flows through very small diameter exhibits unique characteristics like high Knudsen number, low Reynolds number, high surface to volume ratio etc. As a result of this lot of disparities in flow physics happens for a micro shock tube compared with a macro shock tube.

The most important of these effects is the boundary layer becoming large enough and will significantly affects the flow characteristics. Also a shock tube with few micrometers diameter makes the molecular mean free path comparable with the flow dimension. As a result of this inter-molecular force becomes pre dominant in such flows. Many applications require the micro shock tube to operate at low pressure which causes rarefaction effects to happen. Rarefaction effect brings additional velocity and temperature jump to the near wall fluid. These differences in flow physics necessitate a detailed investigation to predict the shock propagation in a micro shock tube.
Shock propagation investigation in micro shock tubes mainly started with the experimental study of Duff $^{(3)}$. He observed that shock propagation suffered a greater attenuation at lower diameters. Brouillette $^{(4)}$ extended this work by conducting experimental studies on shock tube with $5.3 \mathrm{~mm}$ diameter with different initial driven pressure. He observed that the shock attenuation is directly proportional to the initial pressure. He also devised a scaling factor for the generation and propagation of shock waves. Later Zeiton ${ }^{(5,6)}$ carried out computational analysis to simulate the shock propagation under rarefied conditions. Recently Amit ${ }^{(7)}$ studied the sudden expansion and contraction flows in a micro tube.

Many of the past works on this topic did not provided much information on the slip effects in shock propagation. Also not many studies have been done on the post shock reflection flow field under slip conditions. This paper mainly focuses on giving a better explanation for the shock flow field under slip conditions. The effect of diameter and initial pressure on shock propagation was also investigated.

\section{Numerical Simulation}

\subsection{Computational Domain}

A 2D axis-symmetric model of the micro shock tube was considered for the present study as shown in Fig. 1. Micro shock tube consists of a driver and driven section separated by a diaphragm. When the diaphragm breaks the high pressure driver gas escapes to the low pressure driven section producing a moving shock wave.

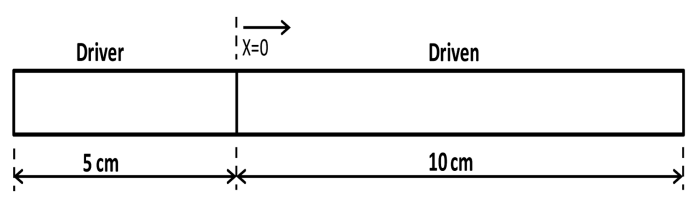

Fig. 1. Schematic diagram of computational domain.

\subsection{Computational Grid}

The computational domain was decomposed into structured quadrilateral cells for the numerical study. The accuracy of numerical prediction heavily depends 
on the grid quality and distribution. So to avoid the numerical errors due to the grid a mesh independent study was carried out with three different mesh distributions $(700 \times 10,1000 \times 20,1500 \times 25)$. The last grid with a size of $1500 \times 25$ was chosen as the optimized grid as it shows lesser discrepancy in results with the previous mesh simulation, which is shown in Fig. 2.

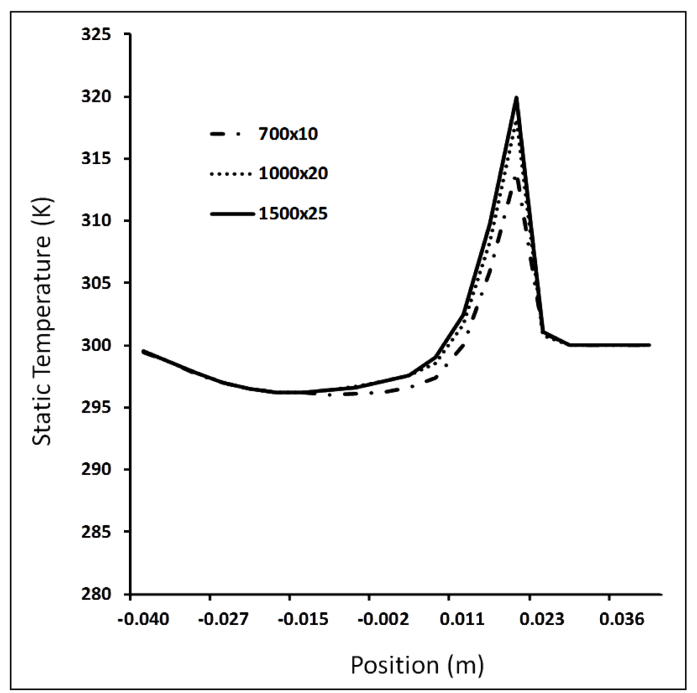

Fig. 2. Temperature distributions (Case B) along axis for different grids at $\mathrm{t}=5 \times 10^{-5} \mathrm{~s}$.

\subsection{Initial Conditions}

For investigating the effect of initial pressure on the shock propagation, a $2 \mathrm{~mm}$ shock tube with helium as driver gas and air as driven gas was numerically simulated. Two different initial pressures with same pressure ratio, as shown in Table 1, were used for this simulation. The effect of slip and no slip conditions on shock propagation were compared for the low pressure cases. All the walls were considered to be isothermal with a temperature of $300 \mathrm{~K}$. Complete rupture of diaphragm was assumed at the starting of the simulation.

Table 1. Initial conditions to study the pressure dependency and slip effects on shock propagation

\begin{tabular}{ccccc}
\hline Case & $\mathrm{P}_{1}(\mathrm{~Pa})$ & $\mathrm{P}_{4} / \mathrm{P}_{1}$ & Diameter & Wall Conditions \\
\hline A & 500 & 15 & $2 \mathrm{~mm}$ & No Slip \\
B & 50 & 15 & $2 \mathrm{~mm}$ & No Slip \\
C & 50 & 15 & $2 \mathrm{~mm}$ & Slip \\
\hline
\end{tabular}

For investigating the shock propagation dependency on the diameter, three different cases were considered with diameter of $2 \mathrm{~mm}, 1 \mathrm{~mm}$ and $0.5 \mathrm{~mm}$ respectively as shown in Table 2.

Table 2. Initial conditions to study the shock propagation dependency on diameter

\begin{tabular}{ccccc}
\hline Case & $\mathrm{P}_{1}(\mathrm{~Pa})$ & $\mathrm{P}_{4} / \mathrm{P}_{1}$ & Diameter & Wall Conditions \\
\hline 1 & 50 & 15 & $2 \mathrm{~mm}$ & Slip \\
2 & 50 & 15 & $1 \mathrm{~mm}$ & Slip \\
3 & 50 & 15 & $0.5 \mathrm{~mm}$ & Slip \\
\hline
\end{tabular}

\subsection{Numerical Schemes}

2D unsteady Reynolds Averaged Navier Stokes equations were used to mathematically model the flow physics. The governing equations in the cartesian form can be written as,

Continuity:

$$
\frac{\partial \rho}{\partial t}+\frac{\partial}{\partial x_{i}}\left(\rho u_{i}\right)=0
$$

Momentum:

$\frac{\partial}{\partial t}\left(\rho u_{i}\right)+\frac{\partial}{\partial x_{i}}\left(\rho u_{i} u_{j}\right)=-\frac{\partial p}{\partial x_{i}}+\frac{\partial}{\partial x_{j}}\left[\mu\left(\frac{\partial u_{i}}{\partial x_{i}}+\frac{\partial u_{j}}{\partial x_{j}}-\frac{2}{3} \delta_{i j} \delta u_{i}\right)\right]$
$+\frac{\partial}{\partial x_{j}}\left(-\rho \overline{u_{i} u_{j}}\right)$

Energy:

$$
\frac{\partial \rho E}{\partial t}+\frac{\partial}{\partial x_{i}}\left[u_{i}(\rho E+p)\right]=\frac{\delta}{\partial x_{i}}\left[\left(\alpha+\frac{C_{p} \mu_{t}}{P_{r t}}\right) \frac{\partial T}{\partial x_{i}}+u_{j}\left(\tau_{i j}\right)_{e f f}\right](3)
$$

$\tau_{\text {eff }}$ is the shear stress tensor which is modeled as,

$$
\left.\tau_{i j}=\mu_{e f f}\left(\frac{\partial u_{j}}{\partial x_{i}}+\frac{\partial u_{i}}{\partial x_{j}}\right)-\frac{2}{3} \mu_{e f f} \frac{\partial u_{j}}{\partial x_{i}} \delta_{i j}\right]
$$

SST-k $\omega$ turbulence model was used to predict the turbulent fluctuations. In this model the turbulent viscosity is calculated by solving the transport equation for turbulent kinetic energy $(\mathrm{k})$ and the specific dissipation rate $(\omega)$ as shown in the below equation.

$$
\begin{aligned}
& \frac{\partial \rho k}{\partial t}+\frac{\partial}{\partial x_{i}}\left(\rho k u_{i}\right)=\frac{\delta}{\partial x j}\left(\Gamma_{k} \frac{\partial k}{\partial x_{j}}\right)+G_{k}-Y_{k} \\
& \frac{\partial \rho \omega}{\partial t}+\frac{\partial}{\partial x_{i}}\left(\rho \omega u_{i}\right)=\frac{\delta}{\partial x j}\left(\Gamma_{\omega} \frac{\partial \omega}{\partial x_{j}}\right)+G_{\omega}-Y_{\omega}+D_{\omega}
\end{aligned}
$$


The mass fraction variation of different species involved was modeled using species transport equations. Second order implicit scheme was used for the time marching. The flux component of the governing integral equation was discretized using AUSM scheme. Third order MUSCL scheme was used to extrapolate the cell center values to the face centers. The governing equations were solved in a coupled manner using commercial solver, Fluent. The low pressure rarefaction effects were modeled using Maxwell's slip boundary ${ }^{(8)}$ equations as given below.

$$
\begin{aligned}
& U_{w}-U_{g}=\left(\frac{2-a_{v}}{a_{v}}\right) \frac{\lambda}{\delta}\left(U_{g}-U_{c}\right) \\
& T_{s}-T_{w}=2\left(\frac{2-a_{T}}{a_{T}}\right) \frac{\lambda}{\delta}\left(T_{g}-T_{c}\right) \\
& \lambda=\frac{k_{B} T}{\sqrt{2} \pi \sigma^{2} p}
\end{aligned}
$$

Here the subscript g, c and $\mathrm{w}$ refer to gas, cell centre and wall quantities respectively. $a_{v}$ and $a_{t}$ are the momentum and thermal accommodation coefficients respectively. $\lambda$ is the molecular mean free path and $\sigma$ represents the L-J characteristics length of gas molecules. $\delta$ is the first layer height.

\section{Results and Discussions}

It is well established that within a shock tube, a shock wave moves into the driven section and an expansion wave moves into the driver section and both being separated by a contact interface. The shock movement inside a micro shock tube of $2 \mathrm{~mm}$ diameter (Case A) is shown in Fig. 3. Here the starting of the temperature jump indicates the contact interface where as the temperature dip position shows the shock front.

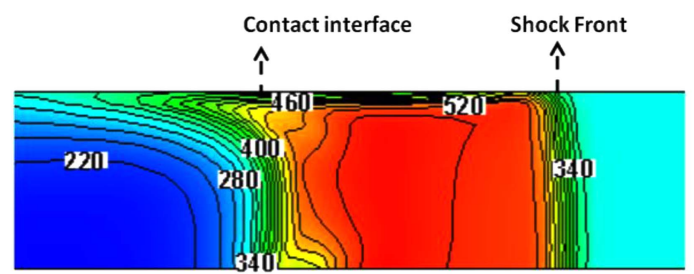

Fig. 3. Temperature distribution (Case A) at $\mathrm{t}=1 \times 10^{-5} \mathrm{~s}$.

The moving shock front position inside a micro shock tube can be analytically determined using the following equation,

$$
\frac{P_{4}}{p_{1}}=\frac{1+\frac{2 \gamma_{1}}{\gamma_{1}+1}\left(M_{s}^{2}-1\right)}{\left[1-\frac{\gamma_{4}-1}{\gamma_{4}+1} \frac{a_{1}}{a_{4}}\left(M_{s}-\frac{1}{M_{s}}\right)\right]^{\frac{2 \gamma_{4}}{\gamma_{4}-1}}}
$$

Here $M_{s}$ is the shock Mach number and $a$ is the sound speed. $P$ and $T$ denote the pressure and temperature respectively.

For the present case, with a pressure ratio of 15 , the theoretical Mach number was found to be 2.34. From the shock Mach number the shock velocity can be easily determined which when multiplied with time gives the theoretical shock location for any particular time. But in real case the shock Mach number does not exhibits a constant value, as stated by the equation, instead it continuously degrades as shock front advances. The reason for this is the viscous losses happening downstream of shock wave which is not taken care by the invicid moving shock equation. It can be observed that a thick boundary layer develops behind the shock front which produces considerable loss in the flow momentum as shown in Fig. 4. Compared to macro shock tube the losses associated with boundary layer for a micro shock tube will be severe due to very small flow diameter.

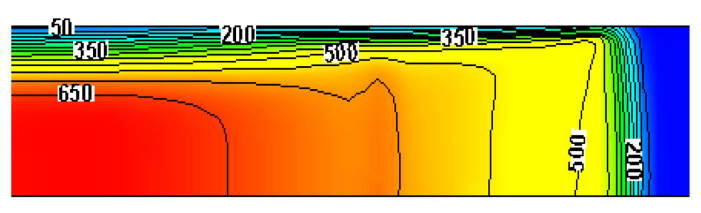

Fig. 4. Axial velocity distribution (Case A) at $\mathrm{t}=1 \times 10^{-5} \mathrm{~s}$.

It can be observed that the boundary layer fills a considerable volume of the domain and core flow is confined to a lesser space.

Another important parameter on which the shock propagation depends is the initial pressure value existing inside the micro shock tube. The simulation with lower initial pressure (Case B) shows a more steep attenuation in shock strength compared with high pressure case (Case A), both being simulated with same pressure ratio and diameter, as shown in Fig. 5.

The reason for this phenomenon is that as the 


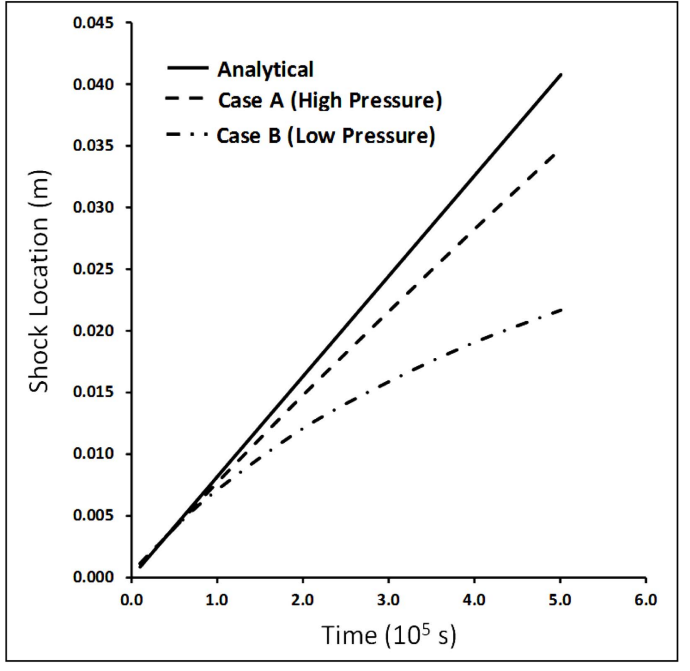

Fig. 5. Shock locations along axis $(d=2 \mathrm{~mm})$ at different initial pressure conditions.

pressure decreases the boundary layer thickness increases. This will increase the viscous losses producing more degradation in shock strength. A comparison of boundary layer formation downstream of shock for high pressure and low pressure, as shown in Fig. 6, clearly explains this.
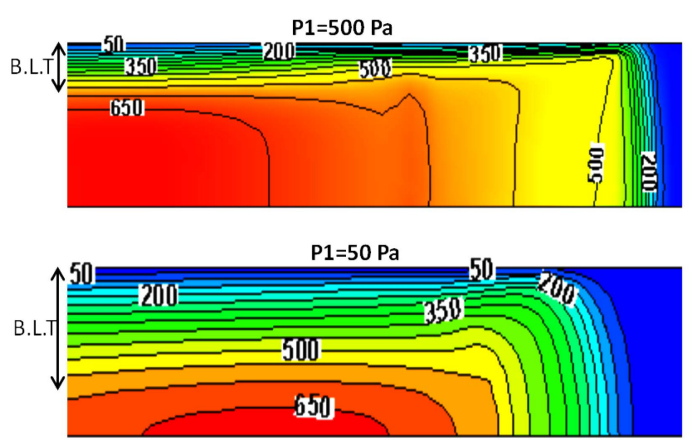

B.L.T $\rightarrow$ Boundary Layer Thickness

Fig. 6. Axial velocity distributions for Case $A\left(P_{1}=500\right.$ $\mathrm{Pa})$ and Case $\mathrm{B}\left(\mathrm{P}_{1}=50 \mathrm{~Pa}\right)$ at $\mathrm{t}=1 \times 10^{-5} \mathrm{~s}$.

The low pressure existing in the system necessitates the inclusion of rarefaction effects to the flow simulation. This brings a finite slip velocity and temperature jump to the wall attached fluid. The shock propagation and its characteristics are affected by this change in the near wall flow field.

With the slip wall boundary conditions the shock front moves faster compared to the no slip wall condition which can be observed from the shock location comparison as shown in Fig. 7. This clearly indicates that the shock attenuation reduces under rarefied conditions.

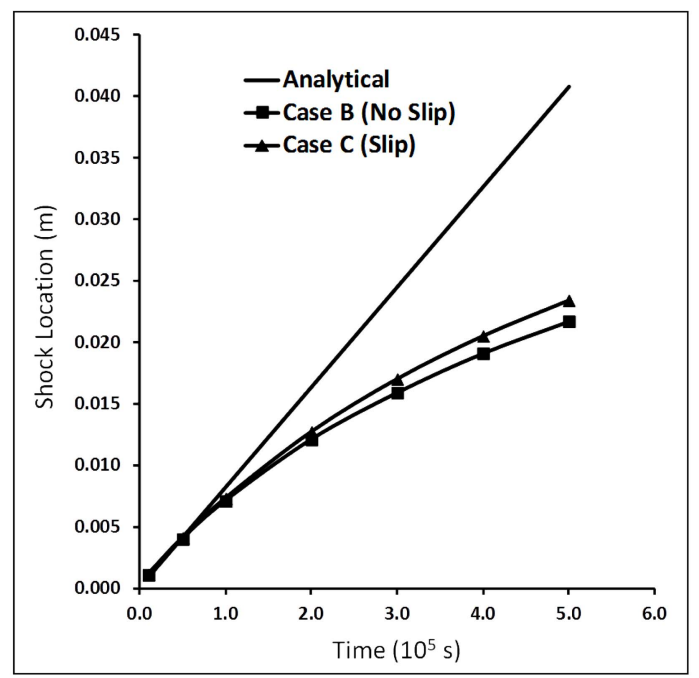

Fig. 7. Shock locations along axis $(\mathrm{d}=2 \mathrm{~mm})$ for slip and no slip cases.

Fig. 8 shows a comparison of temperature field at slip and no slip conditions. It can be clearly observed that both velocity and thermal boundary layer thickness got reduced by the implementation of slip conditions. This produce more pronounced core flow for slip conditions and eventually aids in shock propagation.

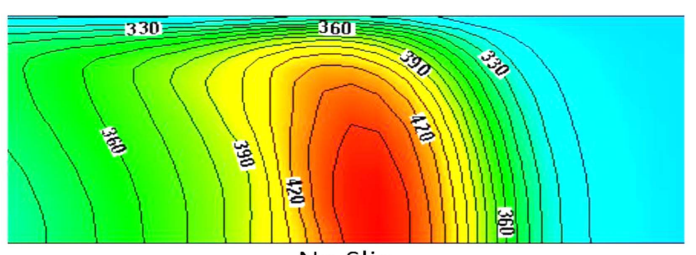

No Slip

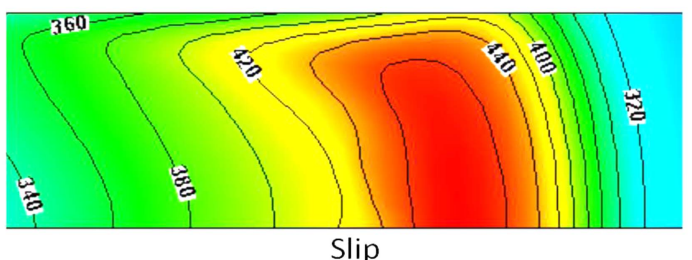

Fig. 8. Temperature distributions for Case B (no slip) and Case $\mathrm{C}(\mathrm{slip})$ at $\mathrm{t}=1 \times 10^{-5} \mathrm{~s}$. 
A comparison of shock position at various times for different diameters is shown in Fig. 9. It can be noticed that the shock speed is drastically reduced as the diameter decreases and the shock wave ceases into a weak compression wave within a short time.

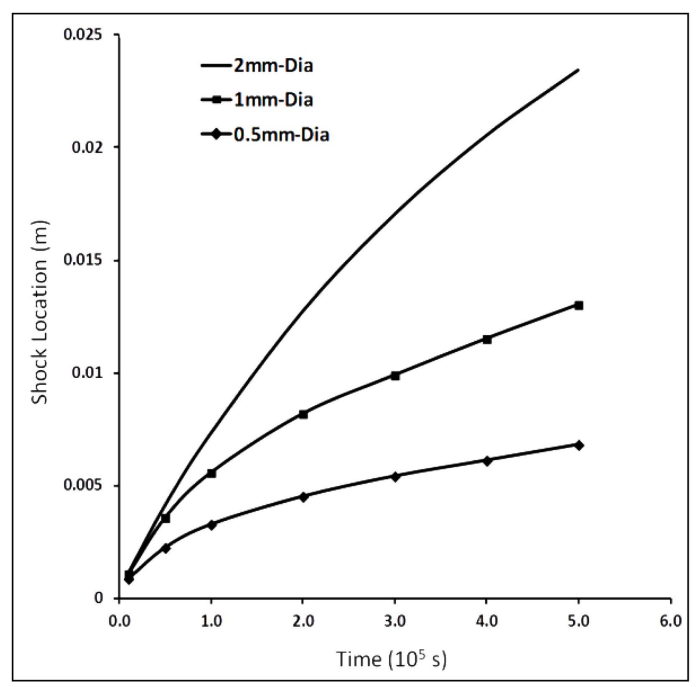

Fig. 9. Shock locations along axis for different tube diameters.

The major reason for this is that as the tube diameter decreases the boundary layer fills up majority of the flow volume and the core flow will be narrowed. The decrease in shock strength can be

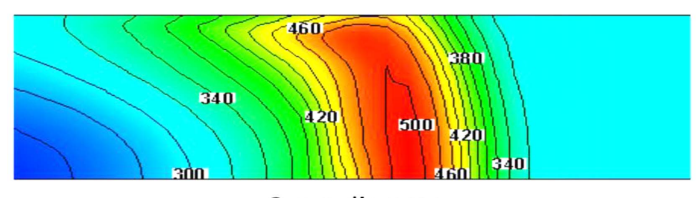

$2 \mathrm{~mm}$ diameter

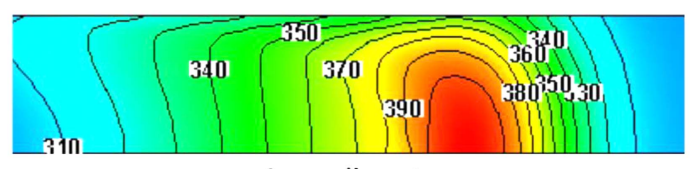

$1 \mathrm{~mm}$ diameter

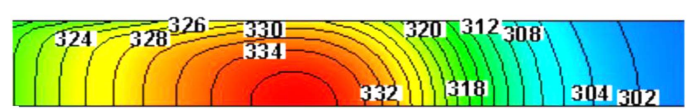

$0.5 \mathrm{~mm}$ diameter

Fig. 10. Temperature distributions for Cases 1,2 and 3 respectively at $\mathrm{t}=1 \times 10^{-5} \mathrm{~s}$. observed from the temperature contour comparison for different diameter cases as shown in Fig. 10.

The reflected shock wave characteristics with slip condition were studied for the $2 \mathrm{~mm}$ shock tube with initial conditions same as Case C. But for this study the tube length was reduced with a driver section of $10 \mathrm{~mm}$ and driven section of $15 \mathrm{~mm}$. The shock reflection causes an increase in pressure downstream of the shock wave. This leads to an increase in Knudsen number behind the reflected shock front which makes the flow there to change back to no slip case. As a result of this more losses happens to the flow and the shock wave gets converted into weak compression wave soon after reflection. The reflected shock position comparison for slip and no slip case is shown in Fig. 11

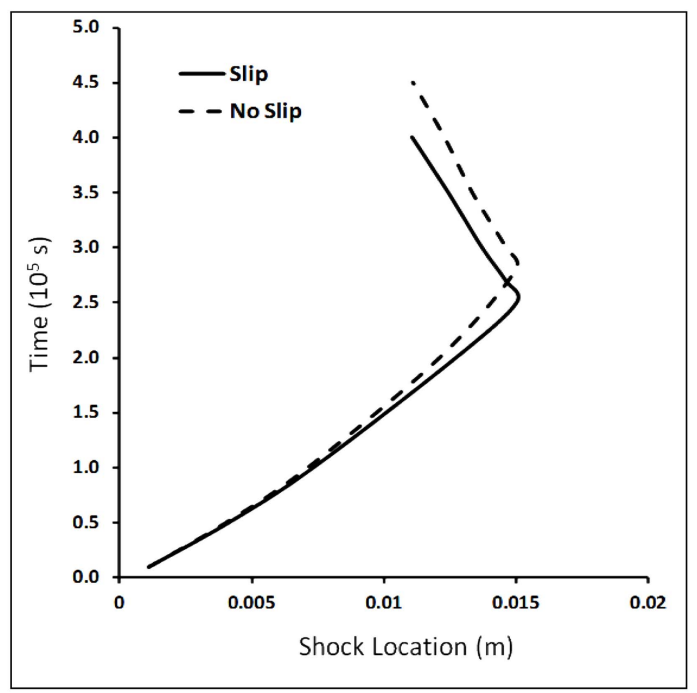

Fig. 11. Reflected shock locations along axis $(\mathrm{d}=2 \mathrm{~mm})$ for Case B (no slip) and Case C (slip).

\section{Conclusion}

Cfd simulation carried out on a micro shock tube reveals that the shock wave suffers greater attenuation compared to a macro shock tube. The major reason for this is the development of thick boundary layer behind the shock front and its associated losses. The boundary layer thickness increases with reduction in pressure. Hence as pressure reduces more losses happen to the flow field and this leads to more severe attenuation of shock strength. The 
lower pressure induces rarefaction effects which will give additional velocity and temperature jump to the near wall fluid. This eventually helps in shock propagation. The shock strength has a strong dependency on tube diameter also. As tube diameter decreases the shock strength degrades severely. This is because the boundary layer fills up majority of the flow volume producing rigorous losses. When the shock wave reflects from the end wall it increases the downstream pressure and if the initial pressure is not sufficiently low there is a huge possibility that the rarefaction effects vanishes and near wall fluid attaches to the wall. This cause additional loss to the flow and the shock wave get transformed into weak compression wave soon after reflection.

\section{Acknowledgement}

This work was supported by the National Research Foundation of Korea (NRF) grant funded by the Korea government (MEST) (2011-0017506).

\section{References}

1) Li Junwei and Zhong Beijing., 2008, "Experimental investigation on heat loss and combustion in methane/oxygen micro-tube combustor", Applied Thermal Engineering., Vol. 28, pp. 707-716.

2) Liu, Y. and Kendall, M.A.F., 2006, "Numerical analysis of gas and micro-particle interactions in a hand-held shock-tube device", Biomed Microdevices., Vol. 8, pp. 341-351.

3) Duff, R.E., 1959, "Shock tube performance at initial low pressure", Phys.Fluids., Vol. 2, pp.207-216

4) Brouillete, M.,2003, "Shock waves at microscales", Shock Waves., Vol. 13, pp. 03-012

5) Zeitoun, D. E., Burtschell, Y., 2006, "Navier- Stokes computations in micro shock tubes", Shock Waves, Vol. 15, pp. 241-246

6) Zeitoun, D. E., Burtschell, Y., Graur, I. A., Ivanov, M. S., Kudryavtsev, A. N., Bondar, Y. A., 2009, Numerical simulation of shock wave propagation in micro channels using continuum and kinetic approaches, Shock Waves, Vol. 19, pp. 307-316

7) Amit Agrawal., Lyazid Djenidi., Antonia, R.A.,2005, "Simulation of gas flow in mcrochannels with a sudden expansion or contraction", Journal of Fluid Mechanics, Vol. 530, pp. 135-144

8) Karniadakis, G. E. M., Beskok, A., 2000, Micro Flows fundamentals and Simulation, Springer, Berlin Heidelberg, New York, pp. 45-62. 